\title{
XLII. New criteria for the imaginary roots of equations
}

\section{J.R. Young Esq.}

To cite this article:J.R. Young Esq. (1843) XLII. New criteria for the imaginary roots of equations, Philosophical Magazine Series 3, 22:145, 252-258, DOI: 10.1080/14786444308636372

To link to this article: http://dx.doi.org/10.1080/14786444308636372

册 Published online: 01 Jun 2009.

Submit your article to this journal $\pi$

Џ Article views: 3

Q View related articles $₫$ 
pass any photographic production I have yet seen, and which indeed it seems impossible to go beyond. Most unfortunately, they cannot be preserved. Every attempt to fix them has resulted in the destruction of their beauty and force; and even when kept from light, they fade with more or less rapidity, some disappearing almost entirely in three or four days, while others have resisted tolerably well for a fortnight, or even a month. It is to an over-dose of tartaric acid that their more rapid deterioration seems to be due, and of course it is important to keep down the proportion of this ingredient as low as possible. But without it $\bar{I}$ have never succeeded in producing that peculiar velvety aspect on which the charm of these pictures chiefly depends, nor anything like the same intensity of colour without over-sunning.

230. I might here describe many other curious and interesting photographic results to which, under the genial influence of such a summer as, possibly, has never before been witnessed in England, I have been conducted. But in so doing I should surpass the reasonable bounds of a Postscript illustrative of my text, and abuse the privilege accorded me. Yet I cannot forbear noticing one at least, in which a line or dot engraving of any degree of delicacy is imitated, line for line, and dot for dot, in a manner which might deceive any but a practised artist to the point of rendering him unable to declare that the photograph had not been struck off from the original plate with common printing ink, by the ordinary process of copper-plate printing. The details of this process, which are delicate and somewhat tedious, cannot properly be stated here; if for no other reason, because I have not yet obtained a complete command over the result : but a microscopic examination of the specimens placed in the hands of our worthy Secretary, though somewhat marred by the accidents of manipulation, will I think suffice to justify the terms employed above.

XLII. New Criteria for the Imaginary Roots of Equations. By J. R. Younc, Esq., Professor of Mathematics in Belfast College.

[Continued from p. 188 and concluded.]

${ }^{r} T$ is easy to see that the foregoing criteria furnish the rules proposed by Newton, in the Universal Arithmetic, for detecting imaginary roots in an equation. These rules have not, I believe, as yet been demonstrated; although, on account of their obvious utility and ready application, a rigorous proof of their truth has frequently been sought. The earliest dis- 
cussion of Newton's rules is that of Maclaurin, in the Philosophical Transactions, vol. xxxvi. No. 408, who has entered into a very long and elaborate investigation of them, the results of which, however, only go the length of showing that " some imaginary roots exist in an equation," whenever any of Newton's criteria have place; and do not embrace the more general affirmation of the rules, that there are as many pairs of such roots as there are distinct criteria fulfilled.

It is no doubt from the misgiving and uncertainty always attendant upon an undemonstrated principle, however numerous the individual instances in which it may have been safely trusted, that these rules of Newton have fallen into neglect, in the analysis of equations. It is one object of the present paper to revive and demonstrate them: another, and the more immediate one, is to prove the adequacy of the criteria already given to determine the true character of a pair of doubtful roots, as soon as by actual development we have reached the point where, if they are real, the separation of them must take place.

It is desirable, when this stage of the approximation is arrived at, that we should be enabled to pronounce at once upon the nature of the roots under examination, from the conditions necessarily impressed upon the transformed coefficients thus attained, without having to apply to additional tests, or to execute any new transformations or by-operations, for this purpose, as in the methods hitherto proposed. This object may be effected from the following considerations.

I have elsewhere shown (Theory of Equations, p. 263), that when two roots, differing but little from equality, or concurring in several leading figures, are to be developed, these figures, after a certain early stage, will be furnished, one after another, by either of the two concurrent expressions which, in the arrangement below, stand vertically under the functions into which these roots first enter:-

$$
\begin{aligned}
& f_{n-2}(x) \ldots \ldots \ldots \ldots f_{2}(x) \quad f_{1}(x) \quad f(x) \\
& \frac{A_{n-1}}{n A_{n}} \quad \ldots \ldots \ldots \ldots \frac{A_{3}}{4 A_{4}} \quad \frac{A_{2}}{3 A_{3}} \quad \frac{A_{1}}{2 A_{2}} \\
& \frac{2 \mathbf{A}_{n-2}}{(n-1) \mathbf{A}_{n-1}} \ldots \ldots . . . \frac{2 \mathbf{A}_{2}}{3 \mathbf{A}_{3}} \quad \frac{\mathbf{A}_{1}}{\mathbf{A}_{2}} \quad \frac{2 \mathbf{A}_{0}}{\mathbf{A}_{1}} \text {. }
\end{aligned}
$$

and further, that when there is a discrepancy between the leading figures furnished by the two expressions used, the roots, if real, are about to separate. Now, without applying to any external source, or extending the development beyond 
the stage thus reached, the character of the discrepancy adverted to will, of itself, decide the doubtful point; for the disagreement may consist either in the first expression giving a greater figure than the second, or the second a greater figure than the first: if the former happen, the roots sought will be real; if the latter, they will be imaginary. For it is plain that in the quadratic, to which the approximation tends (Equations, p. 262), we shall have, for $f(x), n=2$; for $f_{1}(x), n=3$; for $f_{2}(x), n=4$, and so on; so that the conditions previously given supply, in these cases, the following criteria of the character of the roots sought, that is the roots are real or not, according as these conditions exist or fail :-

$$
\begin{aligned}
\frac{A_{1}}{2 A_{2}}>\frac{2 A_{0}}{A_{1}} \\
\frac{A_{2}}{3 A_{3}}>\frac{A_{1}}{A_{2}} \\
\frac{A_{3}}{4 A_{4}}>\frac{2 A_{2}}{3 A_{3}} \\
\vdots \\
\frac{A_{n-1}}{n A_{n}}>\frac{2 A_{n-2}}{(n-1) A_{n-1}} .
\end{aligned}
$$

The value of these criteria, in connexion with the rapid mode of approximation taught in the work referred to, is obvious. In pursuing a pair of contiguous roots of $f_{m}(x)=0$, conformably to that method, we are to seek the development of the intervening root of $f_{m+1}(x)=0$, the successive figures of which, after a step or two, are always furnished by the concurring expressions above, and are to carry on the work up to $f(x)$; continuing the process as long as those expressions agree in giving the same leading figure. When this agreement ceases, the roots may be pronounced real, if the first expression exceed the second; otherwise they will be imaginary. And thus their character unfolds itself spontaneously, without any appeal to external tests or supplementary transformations.

The ultimate quadratic thus attained may with propriety be called the indicator of the doubtful roots; when it proves their reality, we may employ it to supply the leading figures of the two roots, which become distinct after the indicator is reached. This same indicator will also furnish an approximation even to the remaining imaginary portion of the par- 
tially developed roots, when an imaginary pair is indicated, provided $f_{m}(x)$ has approximated closely to zero.

Moreover, when three roots concur in several leading figures, we should in like manner arrive at a cubic indicator; and when there are four such roots, at an indicator of the fourth degree; and so on. And these indicators, like as in the quadratic, would point out the initial directions which the separated roots take. But it is unnecessary to examine these indicators of the higher orders, all of which are ultimately reducible to quadratics: so that in examining minute intervals, in the theory of equations, like as in discussing the elements of a curve surface, the quadratic indicator is sufficient to supply, in both cases, all the desired information.

I shall now return to the general criteria at first given, and shall show their importance in detecting imaginary roots, previously to any actual development, and solely from an examination of the proposed coefficients; and shall thence deduce the rules of Newton before adverted to.

It is well known that for the purpose of examining into the character of the roots of an equation, as to real and imaginary, we may replace that equation by a series of limiting equations of inferior degree: as for instance, if the equation be above the third degree, by a series of cubic equations; or, if we please, by a series of quadratics. In the present inquiry it will be proper to: take the limiting cubics, and not the quadratics, as Maclaurin, and all other investigators of Newton's rule have, I believe, done.

If any of these limiting cubics indicate imaginary roots, such indications will of course also imply imaginary roots in the proposed equation. But several indications, apparently distinct, may offer themselves in these equations, which upon closer examination may be found to be necessarily dependent, and thus to concur in pointing to but a single imaginary pair. Distinct imaginary pairs can be inferred only from distinct independent conditions: we have therefore to inquire how these are to be discovered in the series of limiting equations alluded to.

1. And first we may remark that a cubic equation consists of only four terms; and as but one imaginary pair can enter into it, it follows that whether the criterion at page 186 hold with respect to the three leading terms, or with respect to the three final terms, or in reference to both sets of three, one imaginary pair, and one only, is implied.

2. The cubics we are considering are so connected together, that if the criterion hold, or fail, in reference to the three leading terms of one, it must of necessity, in like manner, hold, 
or fail, in reference to the three final terms of that next in order.

Hence the condition holding for the three leading terms of one cubic necessitates its holding for the three final terms of the next, so that the concurrence implies but a single imaginary pair. By examining our series of cubics, with these principles before us, applying the test to each group of three terms in succession, we shall obviously be able to distinguish those conditions which are really distinct and independent, from those that are not, and therefore to infer so many distinct imaginary pairs.

If the first set of three, that is the leading terms in the first cubic, satisfy the criterion, we immediately infer the existence of one imaginary pair; if the next set-the final terms of the same cubic-also satisfy it, the preceding condition merely recurs, and supplies no new information. In this case the following set of three-the leading terms of the next cubicmust furnish the same concurring condition, by the second principle above; and so on, till we arrive at a set of three for which the criterion fails, thus putting a stop to the series of concurring conditions, and preparing the way for new and independent conditions. As soon as the criterion again holds, the condition, being altogether independent of the former, must imply another and distinct imaginary pair; and so on, to the end of the series.

Now the criteria which we have here supposed to be applied to the terms, taken three at a time, of the successive limiting cubics, supply one after another the very expressions that we have exhibited at page 187; the three final terms of one cubic always furnishing the same expressions as the three leading terms of the next, as noticed above. Consequently, without the formal intervention of the limiting cubics, which have merely been introduced into the reasoning for the purpose of tracing the dependent conditions, we may at once apply the criteria (page 187) to the coefficients of the proposed equation, observing that when the condition recurs, in proceeding from one set of three to the next, the recurrence is to be regarded merely as a repetition of the same indication; that as soon as it fails, preparation is made for the occurrence of a new indication, and so on.

Hence the indications that are really independent, and consequently the number of imaginary roots inferrible from them, may be thus noted. Under the first and last terms of the proposed equation write the sign plus; then, taking each of the intermediate terms in succession for a middle term, write under it the sign minus when the criterion holds, and plus 
when it fails: the alternations of sign, thus furnished, will denote the number of imaginary roots, at least, which enter the equation : and this is Newton's rule.

The rule now established will be found a valuable adjunct in the modern theory of numerical equations; more especially in connexion with the researches of Fourier. We shall apply it to an example taken from the Analyse des Equations of this anthor:-

$$
\begin{aligned}
& x^{5}+x^{4}+x^{3}-2 x^{2}+2 x-1=0 \\
& +-+-+
\end{aligned}
$$

Hence the equation has four imaginary roots. In the work from which this example is taken, a good deal of labour is expended upon arriving at this conclusion.

To what has now been done it may be proper to add, that although the criteria at page 187 have been deduced from Sturm's theorem, yet they may be readily inferred from independent principles; and, moreover, without any direct reference to the limiting equations of Newton and Maclaurin, adverted to above. For it is shown in the Theory of Equations, $p .323$, that if the general equation

$$
\mathrm{A}_{n} x^{n}+\mathrm{A}_{n-1} x^{n-1}+\mathrm{A}_{n-2} x^{n-2}+\ldots \ldots \ldots \ldots \mathrm{A}_{0}=0
$$

be transformed into another, by substituting $x+r$ for $x$, then the third coefficient of the transformed equation, in order that the second may vanish, must be

$$
\frac{A_{n-2}}{A_{n}}-\frac{n(n-1)}{2}\left\{\frac{A_{n-1}}{n A_{n}}\right\}^{2}
$$

Consequently if, when this evanescence takes place, the expression here written have the same sign as $A_{n}$, the zero, then occurring between like signs, will indicate imaginary roots. Hence, multiplying by the positive quantity $2 n \mathrm{~A}_{n}{ }^{2}$, two imaginary roots will be indicated provided we have the condition

$$
2 n A_{n-2} A_{n}>(n-1) A_{n-1}^{2}
$$

or, by reversing the coefficients, provided we have the condition

$$
2 n \mathrm{~A}_{0} \mathrm{~A}_{2}>(n-1) \mathrm{A}_{1}{ }^{2}
$$

and this, applied to the final terms of the successive derived equations, will obviously furnish the series of criteria at p. 187. As imaginary roots are equally indicated though the third coefficient actually vanish along with the'second-except all the roots are equal to $r-$ it follows, as at p. 186, that the sign of inequality may be changed into that of equality without disturbing the indications.

In terminating these investigations, I may perhaps be perPhil. Mag. S. 3. Vol. 22. No. 145. April 1843. 


\section{The Rev. Brice Bronwin on M.Jacobi's Theory}

mitted to remark, that several interesting inquiries of a kindred nature are suggested by them: the prosecution of these I propose to publish in $\approx$ distinct form, as a supplement to the volume already referred to.

J. R. Young.

XLIII. On M. Jacobi's Theory of Elliptic Functions. By the Rev. Brice Bronwin*.

IN page 36 of his Fundamenta Nova, \&c., M. Jacobi, making $\omega=\frac{m \mathrm{~K}+m^{\prime} \mathrm{K}^{\prime} \sqrt{-1}}{n}$, says that $m$ and $m^{\prime}$ may be any

integer numbers, positive or negative, provided they have no common factor, which also measures $n$. What $I$ intend in this paper is, to prove that $m$ must be an odd and $m^{\prime}$ an even number, and that no other form is admissible. If $r$ and $r^{\prime}$ be integers, positive or negative, the value of $\omega$, as above defined, includes the four following forms:-

$\omega=\frac{(2 r+1) \mathrm{K}+2 r^{\prime} \mathrm{K}^{\prime} \sqrt{-1}}{n}, \sin a m n \omega= \pm 1, \cos a m n \omega=0$.

$\omega=\frac{2 r \mathrm{~K}+2 r^{\prime} \mathrm{K} \sqrt{-1}}{n}, \sin a m n \omega=0, \cos a m n \omega= \pm 1$.

$\omega=\frac{2 r \mathrm{~K}+\left(2 r^{\prime}+1\right) \mathrm{K}^{\prime}}{n} \sqrt{-1}, \sin a m n \omega= \pm \infty \sqrt{-1}, \cos a m n \omega= \pm \infty$.

$\omega=\frac{(2 r+1) \mathrm{K}+\left(2 r^{\prime}+1\right) \mathrm{K}^{\prime} \sqrt{-1}}{n}, \sin a m n \omega= \pm \frac{1}{k}, \cos a m n \omega= \pm \frac{k^{\prime}}{k} \sqrt{-1}$.

The values of $\sin a m n \omega, \cos a m n \omega$ are arnexed on account of their importance in what follows. They are calculated by the formula at pages 32 and 34 . The references here made are all to the Fundamenta Nova, and the notation adopted there is employed here, unless express mention be made to the contrary. But to abridge I shall write $\mathrm{s} a u$ for $\sin a m u, \mathrm{c} a u$ for $\cos a m u, \& \mathrm{c}$.

At page 38 we have for the general transformation, $\mathrm{s} a \frac{u}{\mathrm{M}}=\mathrm{Cs} a u \mathrm{~s} a(u+4 \omega) \mathrm{s} a(u+8 \omega) \ldots \mathrm{s} a(u+4(n-1) \omega), .(1$. or

$\mathrm{s} a \frac{u}{\mathrm{M}}=\frac{\frac{x}{\mathrm{M}}\left(1-\frac{x^{2}}{\mathrm{~s}^{2} a 4 \omega}\right)\left(1-\frac{x^{2}}{\mathrm{~s}^{2} a}\right) \cdots\left(1-\frac{x^{2}}{\left(1-k^{2} x^{2} \mathrm{~s}^{2} a 4 \omega\right)\left(1-k^{2} x^{2} \mathrm{~s}^{2} a 8 \omega\right) \ldots\left(1-k^{2} x^{2} \mathrm{~s}^{2} a 2(n-1) \omega\right.}\right)}{(n-1) \omega)}$.

Here $\frac{1}{C}$ is put for the constant denominator of the second member of (1.) The middle factor of that member is * Communicated by the Author. 